
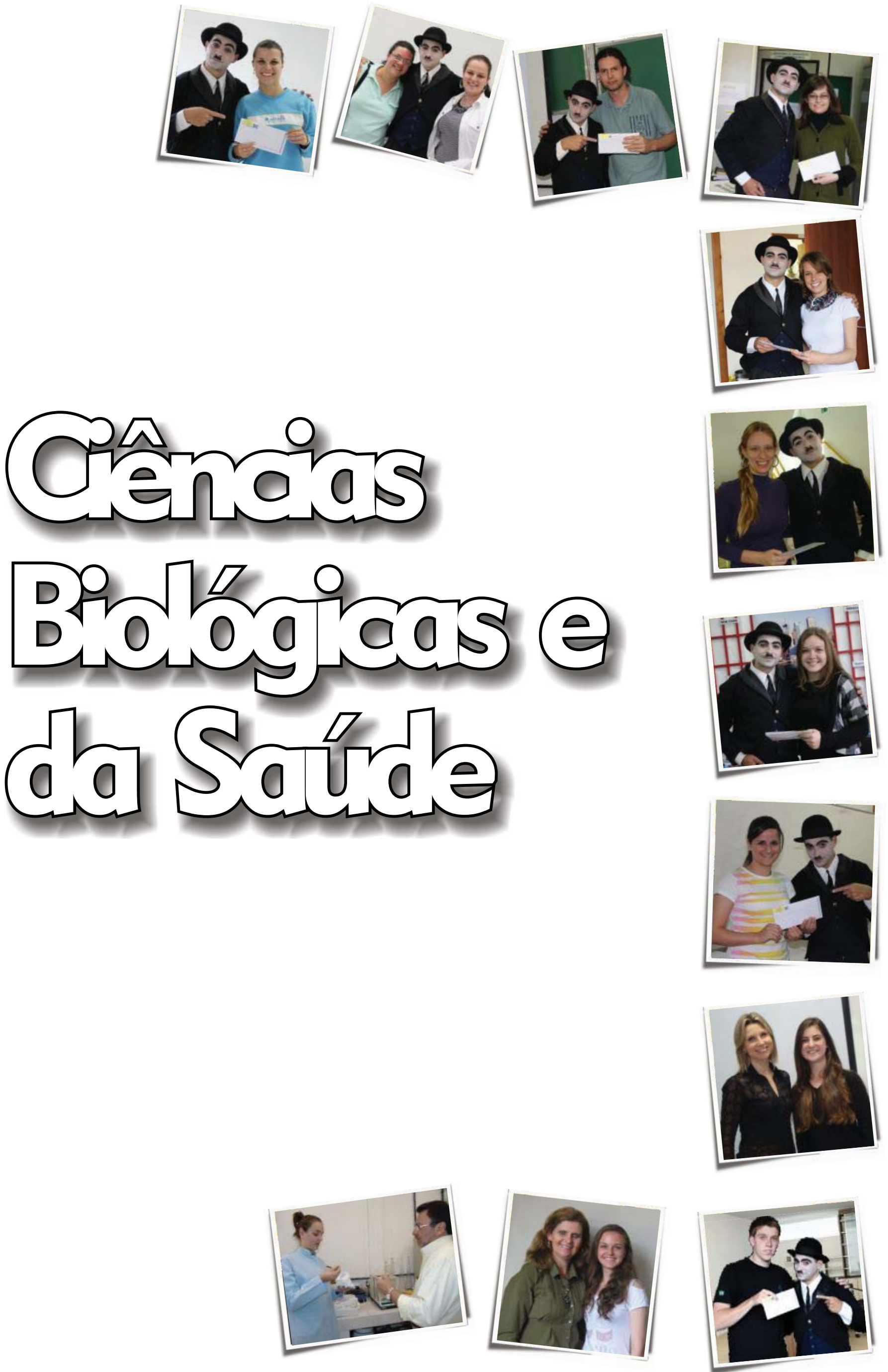


\section{ÁREA DE CIÊNCIAS BIOLÓGICAS E DA SAÚDE}

Na área de Ciências Biológicas e da Saúde entre os 72 trabalhos apresentados no evento, 64 foram de alunos bolsistas de Iniciação Científica da Universidade e 08 trabalhos de alunos de Iniciação Científica vinculados a outras Instituições de Ensino do Estado do Rio Grande do Sul. A maioria dos trabalhos nesta área foi de bolsistas do Programa UNISC de Iniciação Científica - PUIC e de Programas de bolsas como o de verba externa para pagamentos de bolsas em projetos de pesquisa. Também foram apresentados trabalhos contemplados com bolsas de IC do CNPq e da FAPERGS. Importante destacar a participação de estudantes participantes do Programa PUIC voluntário, dados apresentados na Figura 02.

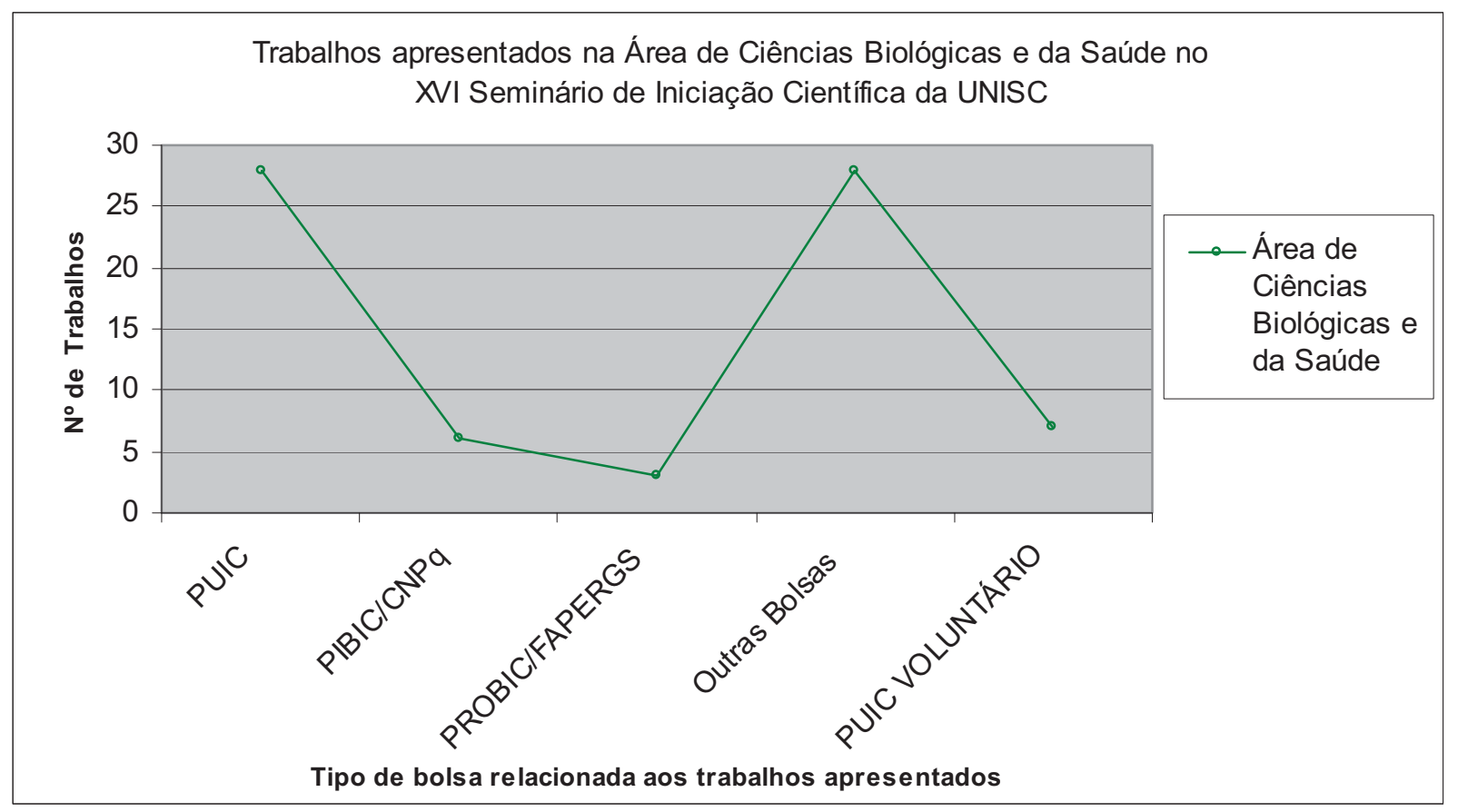

Figura 02 - Modalidade de bolsas dos estudantes participantes do XVI Seminário de Iniciação Científica na Área de Ciências Biológicas e da Saúde.

Fonte: Coordenação de Pesquisa, UNISC, 2010. 


\title{
ANÁLISE DO PERFIL DE MORTALIDADE POR DOENÇA PULMONAR OBSTRUTIVA CRÔNICA NO MUNICÍPIO DE SANTA CRUZ DO SUL, RS, BRASIL
}

\author{
Gabriela Crestani ${ }^{1}$ \\ William Rutzen ${ }^{2}$ \\ Andréa Lúcia Gonçalves da Silva ${ }^{3}$ \\ Tânia Cristina Malezan Fleig ${ }^{4}$ \\ Marcelo Tadday Rodrigues ${ }^{5}$
}

\section{RESUMO}

Contextualização: A Doença Pulmonar Obstrutiva Crônica (DPOC) é caracterizada funcionalmente por limitação ao fluxo aéreo que não é totalmente reversível. 0 tabagismo é o principal fator de risco. A DPOC era a sexta causa de morte no mundo em 1990, segundo a OMS, com estimativa para tornar-se a terceira até 2020, sendo que vários componentes contribuem para o crescente aumento na mortalidade por tal doença, como aumento do tabagismo pelas mulheres, aumento da expectativa de vida, entre outros. Objetivo: Analisar o perfil da mortalidade por DPOC no município de Santa Cruz do Sul por DPOC, utilizando uma estimativa de dois anos consecutivos (2007 e 2008), verificando o perfil dos pacientes como sexo, idade, presença de comorbidades, se faleceu em ambiente hospitalar ou domiciliar. A intenção do estudo constitui também uma comparação com os dados presentes na literatura. Método: Delineamento transversal, levantamento epidemiológico via análise dos registros de óbitos ocorridos entre os períodos de 01 de Janeiro de 2007 a 31 de Dezembro de 2008 na cidade de Santa Cruz do Sul, obtidos através da Vigilância Epidemiológica, registrados tendo como causa-base o DPOC (J44) segundo a Classificação

1 Acadêmica do curso de Medicina, departamento de Educação Física e Saúde da Universidade de Santa Cruz do Sul - UNISC, Rio Grande do Sul - Brasil. [gabicrestani@yahoo.com.br]

2 Acadêmico do curso de Medicina, departamento de Educação Física e Saúde da Universidade de Santa Cruz do Sul - UNISC, Rio Grande do Sul - Brasil. [williamrutzen@yahoo.com.br]

3 Coordenadora do Projeto de Pesquisa Reabilitação Cardiorrespiratória e Metabólica e suas Interfaces, Departamento de Educação Física e Saúde da Universidade de Santa Cruz do Sul UNISC, Rio Grande do Sul - Brasil. [andreag@unisc.br]

4 Coordenadora do Projeto de Pesquisa Reabilitação Cardiorrespiratória e Metabólica e suas Interfaces, Departamento de Educação Física e Saúde da Universidade de Santa Cruz do Sul UNISC, Rio Grande do Sul - Brasil. [tfleig@unisc.br]

5 Coordenador do Projeto de Pesquisa Reabilitação Cardiorrespiratória e Metabólica e suas Interfaces, Departamento de Educação Física e Saúde da Universidade de Santa Cruz do Sul UNISC, Rio Grande do Sul - Brasil. [marcelotadday@unisc.br] 
Internacional de Doenças, 10a edição. Resultados: Ocorreram, no período estudado, 113 óbitos devido a DPOC. Desses, a maioria corresponde ao sexo masculino $(65,48 \%)$, sendo que a média de idade entre os pacientes foi 74,48 anos. Um percentual de 77,87 dos óbitos ocorreu em ambiente hospitalar e do total $94,69 \%$ possuíam duas ou mais comorbidades. Conclusão: Os dados obtidos com a presente análise epidemiológica permite concluir que, na cidade de Santa Cruz do Sul, o perfil dos pacientes que falecem por DPOC possuem características semelhantes aos encontrados na literatura. Vale ressaltar que tal cidade estudada possui grande parte da economia baseada na produção de tabaco, uma vez que várias empresas fumageiras encontram-se instaladas na região, fato que de certa forma contribui para os índices de tabagistas no município. A maior incidência do sexo masculino e a média de idade elevada refletem, entre outros fatores, um maior consumo de tabaco pelos homens e a característica crônica e progressiva da doença.

Palavras-chave: DPOC. Mortalidade. Óbito.

\section{ABSTRACT}

Background: Chronic Obstructive Pulmonary Disease (COPD) is characterized by airflow limitation that is not fully reversible. Smoking is the main risk factor. COPD was the sixth leading cause of death worldwide in 1990, according to WHO, expected to become the third until 2020, with several components contribute to the continued improvement in mortality from this disease, such as increased smoking by women, increased life expectancy, among others. Objective: To assess the mortality from COPD in Santa Cruz do Sul in COPD patients, using an estimate of two consecutive years (2007 and 2008), checking patients' profile such as gender, age, comorbidities, has died in environment hospital or at home. The intent of the study is also a comparison with data from the literature. Method: Cross-sectional epidemiological survey via analysis of records of deaths that occurred between the periods from 01 January 2007 to December 31, 2008 in Santa Cruz do Sul, obtained from the Surveillance, recorded as having the basic question COPD (J44) from the International Classification of Diseases, 10th edition. Results: There were, during the study period, 113 deaths due to COPD. Of these, the majority are male $(65.48 \%)$, and the mean age of patients was 74.48 years. A percentage of 77.87 of the deaths occurred in hospitals and the total $94.69 \%$ had two or more comorbidities. Conclusion: The data obtained from this epidemiological analysis shows that the city of Santa Cruz do Sul, the profile of patients who die from COPD have features similar to those found in the literature. It is noteworthy that this city has studied much of the economy based on tobacco production, tobacco since many are located in the region, a fact that somehow contributes to the rate of smokers in the municipality. The higher incidence in males and high mean age reflect, among other factors, higher tobacco consumption by men and the characteristic chronic and progressive disease.

Keywords: COPD. Mortality. Death. 


\section{INTRODUÇÃO}

A Doença Pulmonar Obstrutiva Crônica (DPOC) era a sexta causa de morte no mundo em 1990, segundo a Organização Mundial da Saúde (OMS), com estimativa para tornar-se a terceira causa morte até 2020. O maior fator de risco para seu desenvolvimento é sabidamente o tabagismo, seguido da inalação de agentes agressores de origem ambiental. Santa Cruz do Sul é considerada a cidade capital nacional do tabaco no beneficiamento de fumo, e também possui indústria de fabricação de cigarros. Desta forma, sua economia possui fortes bases nas fumageiras aqui instaladas, as quais são grande fonte de emprego local e de incentivo ao tabagismo.

O presente trabalho visa a relatar um estudo de caráter analítico e epidemiológico, realizado com pesquisa de dados em Santa Cruz do Sul - RS. O assunto abordado corresponde a DPOC, mais especificamente sobre o perfil de mortalidade por tal doença nesta cidade.

\section{FUNDAMENTAÇÃO TEÓRICA}

A DPOC é uma doença crônica, de caráter inflamatório, com limitação do fluxo aéreo não totalmente reversível. É considerada a sexta causa de óbito no mundo, sendo a única cuja prevalência continua em crescimento. A DPOC é representada pela bronquite crônica e pelo enfisema pulmonar e é uma importante causa de morbidade e mortalidade no mundo moderno. Os pacientes portadores da doença apresentam as características de obstrução de vias aéreas e destruição das paredes alveolares com consequente hiperinsuflação pulmonar (GOLD, 2006). Devido ao caráter progressivo e incapacitante da doença, esta acaba por acarretar um considerável impacto econômico e social, pela redução na produtividade, comprometimento do orçamento familiar, aposentadorias precoces e alto custo com o tratamento e com as internações, que são muito frequentes (FERNANDES et al., 2006).

Acerca dos fatores envolvidos na patogênese da DPOC, o tabagismo é responsável por mais de $90 \%$ dos casos. Outros fatores correspondem a poeiras tóxicas como produtos químicos ocupacionais e combustão de biomassa. 0 tabagismo é o maior responsável pela ocorrência de doencas respiratórias, desde o efeito da exposição da criança "in útero" ou nos primeiros anos de vida, até a vida adulta. A DPOC e o câncer de pulmão destacam-se pelo quadro de progressividade e incapacidade, apesar de terem um maior tempo de latência em relação às doenças cardiovasculares relacionadas ao consumo de tabaco (ARAÚjO, 2009).

Ambos os componentes, enfisema pulmonar e bronquite crônica, estão presentes em proporções variáveis na maioria dos pacientes. A maior resistência ao fluxo aéreo durante a expiração, decorrente da relação inversa e exponencial entre o diâmetro da via aérea e a resistência ao fluxo aéreo, faz com que ocorram aprisionamento aéreo e hiperinsuflação. Outra característica importante é o componente sistêmico da enfermidade, e sabe-se que a inflamação sistêmica nos 
pacientes com DPOC pode estar mais relacionada com a própria doença do que com exacerbações agudas (MATTOS et al., 2009; MULLER et al., 2006 ).

De acordo com dados do Departamento de Informação e Informática do Sistema Único de Saúde (DATASUS), o número de internações hospitalares por DPOC no Brasil no período de 1998 a 2003 foi de 1.480.881, sendo a Região Sul responsável por 42,3\% delas (FERNANDES et al., 2006). O diagnóstico da doença é feito através da história clínica, com identificação de sintomas característicos da DPOC (dispnéia crônica e progressiva, tosse, e produção de muco) bem como a análise de exames de imagem (Raios- $X$ de tórax), laboratoriais e prova de função pulmonar, ou seja, a espirometria com teste com broncodilatador.

O tabagismo deve ser devidamente interrogado e a quantificação de maços/ano é um dado importante a ser obtido. No Brasil, 200 mil mortes anuais são causadas pelo tabagismo e cerca de $16 \%$ da população brasileira adulta é fumante. Ressaltese que em torno de $15 \%$ dos indivíduos que fumam um maço/dia e $25 \%$ daqueles que fumam mais de um maço/dia desenvolvem DPOC (ARAÚJO, 2009; INCA, 2009).

\section{METODOLOGIA}

Estudo transversal e retrospectivo caracterizou-se pelo levantamento epidemiológico via análise dos registros de óbitos. Os dados necessários para a realização deste trabalho foram adquiridos na Vigilância Epidemiológica de Santa Cruz do Sul-RS, utilizando dados registrados tendo como causa-base a DPOC (J44), segundo a Classificação Internacional de Doenças, 10a edição. Foram analisados arquivos com dados de 01 de janeiro de 2007 a 31 de dezembro de 2008, com exceção dos óbitos fetais.

Dentre os dados analisados, os itens utilizados para avaliar o perfil de óbitos por DPOC correspondem à idade, sexo, presença de comorbidades e o local do óbito, se ocorreu em nível hospitalar ou não.

\section{RESULTADOS}

No período de janeiro de 2007 a dezembro de 2008, ocorreram 1987 mortes, considerando excluídos os óbitos fetais. Deste total, 113 ocorreram por DPOC, com uma prevalência de $5,68 \%$. Quando analisados os gêneros, constatou-se um número de 74 óbitos do sexo masculino e 39 do sexo feminino $(65,48 \%$ e $34,52 \%$ respectivamente). 


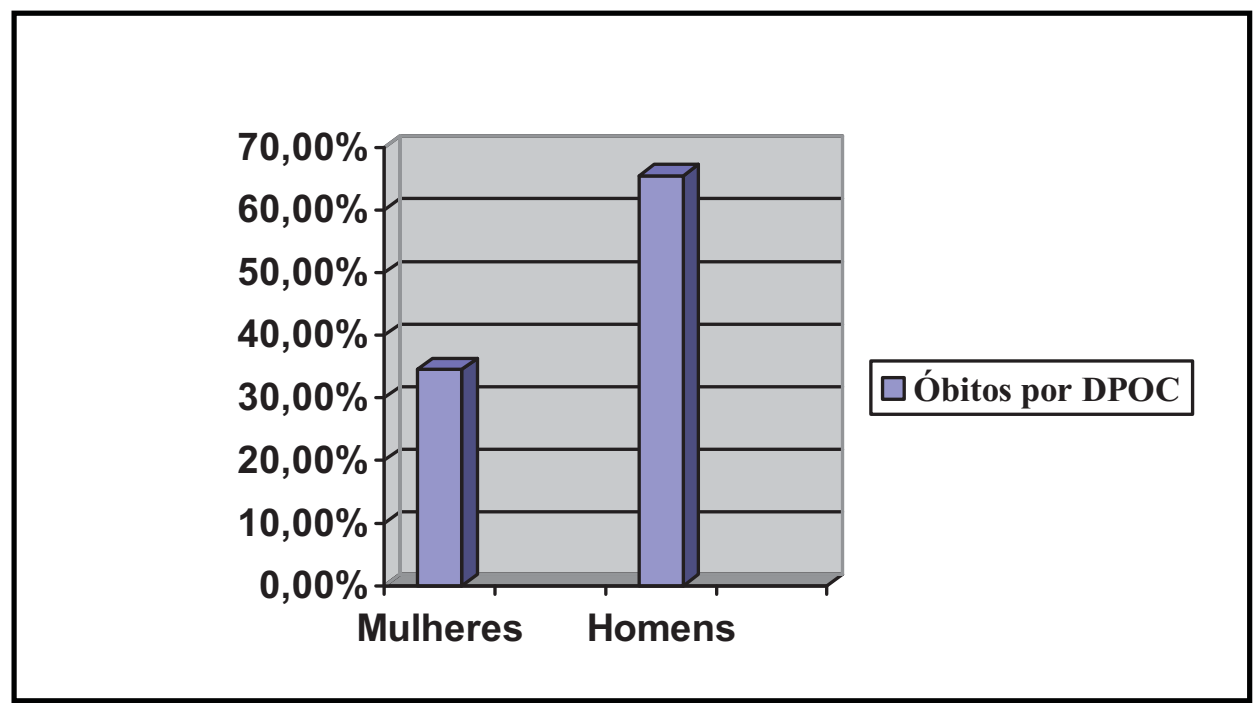
Sul/RS

Gráfico 01 - Óbitos segundo o gênero por DPOC em 2007 e 2008 em Santa Cruz do

Fonte: dados da pesquisa, 2010.

A média de idade constatada foi de 74,48 anos. Dentre os óbitos ocorridos no período estudado, $77,87 \%$ ocorreram em nível hospitalar, num total de 88 falecimentos. Os $22,13 \%$ restantes dos óbitos ocorreram fora do ambiente hospitalar, sendo assim 25 óbitos aconteceram em nível ambulatorial. Em relação às comorbidades as quais os pacientes possuíam, verificou-se que dos 113 óbitos, 107 possuíam duas ou mais comorbidades - $94,69 \%$.

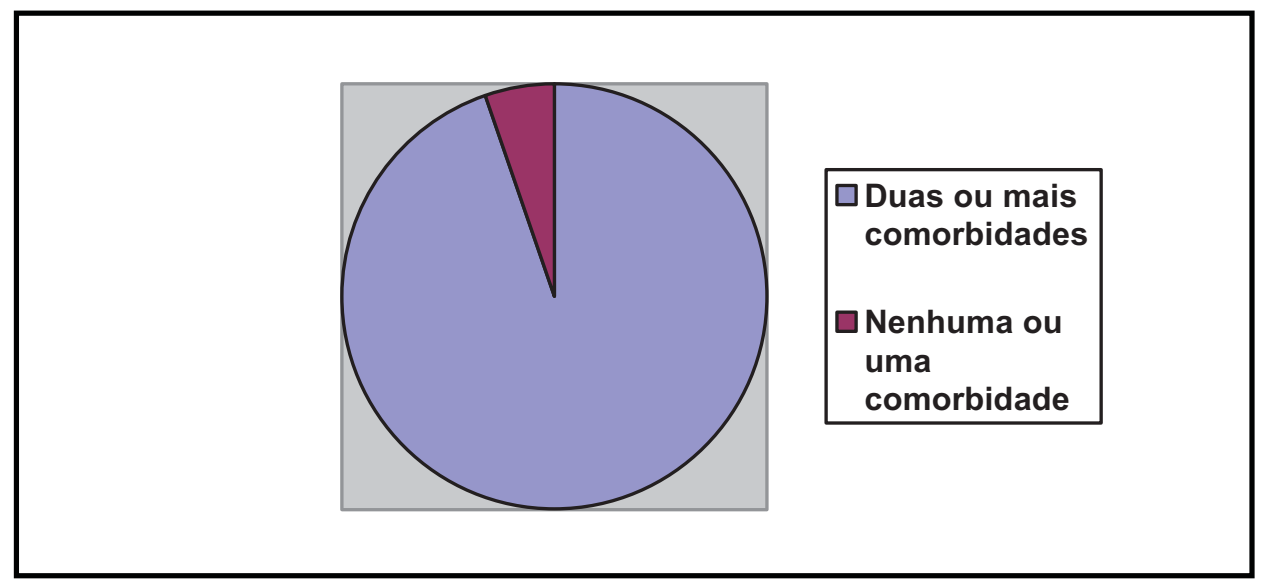

Figura 01 - Presença de comorbidades no momento do óbito

Fonte: dados da pesquisa, 2010.

\section{CONCLUSÃO}

Os dados obtidos através desta análise demonstram uma relação de concordância com o que está na literatura. O presente estudo constatou que os homens falecem mais por DPOC do que as mulheres, podendo tal fato ser justificado 
pelo hábito do tabagismo ser mais comum nesta população. Dados do Instituto Nacional do Câncer (INCA, 2009) afirmam que os homens apresentam aproximadamente $\mathrm{o}$ dobro da prevalência de tabagismo quando comparados às mulheres.

A média de idade avançada dos pacientes cujo óbito ocorreu em vista da DPOC também se relaciona com os estudos prévios. O caráter crônico e progressivo da doença e a demora para o aparecimento dos sintomas, após a exposição ao tabaco, são fatores contribuintes para tal característica. Há estudos que afirmam que a incidência é maior em homens do que em mulheres e aumenta acentuadamente com a idade (GODOY et al., 2007).

O índice maior de mortes no gênero masculino, por DPOC, está condizente com a literatura revisada. As explicações provavelmente estão no fato do maior índice de fumantes do sexo masculino em comparação ao feminino.

O tabagismo é a principal causa evitável de mortes prematuras no Reino Unido e possui grande associação com Doença Pulmonar Obstrutiva Crônica (DPOC), várias formas de câncer e doenças vasculares crônicas, tais como acidente vascular cerebral e doenças coronarianas (MOORE et al., 2009).

A cidade em estudo possui um perfil particular, pois grande parte de sua economia está diretamente relacionada ao principal fator desencadeante da DPOC, o tabaco. Nos indivíduos que desenvolvem a doença, há uma longa exposição ao tabagismo até $o$ aparecimento de sintomas e sinais clínicos da DPOC, assim a média de idade caracteristicamente se eleva.

Assim sendo, fica evidenciado o comprometimento pulmonar e sistêmico da DPOC, e com isso, medidas de controle ao tabagismo devem ser insistentemente mantidas e inovadas. A mortalidade por DPOC afeta a população em geral, e o perfil dos indivíduos que falecem por tal doença em Santa Cruz do Sul segue o mesmo perfil do que é evidenciado na literatura.

\section{REFERÊNCIAS}

GOLD - The Global initiative for chronic Obstructive Lung Disease, 2006

FERNANDES, Amanda et al.Terapia nutricional na doença pulmonar obstrutiva crônica e suas complicações nutricionais. J. bras. pneumol., São Paulo, v.32, n.5, p. 461-471, set./out. 2006.

MATTOS, Waldo Luís Leite Dias de et al. Acurácia do exame clínico no diagnóstico da DPOC. J. bras. pneumol., São Paulo, v. 35, n. 5, p. 404-408, mai. 2009 .

INSTITUTO NACIONAL DO CÂNCER - INCA, 2009. Disponível em: <www.inca.gov.br>. Acesso em: 14 dez. 2010. 
GODOY, Ilda et al. Programa de cessação de tabagismo como ferramenta para o diagnóstico precoce de doença pulmonar obstrutiva crônica. J. Bras. Pneumol. São Paulo, v.33, n.3, p. 282-286, mai-jun. 2007.

ARAÚjO, Alberto José. Tratamento do tabagismo pode impactar a DPOC. Pulmão RJ - Atualizações Temáticas. Rio de Janeiro, 2009.

MULLER, Beat et al. Biomarkers in Acute Exacerbation of Chronic Obstructive Pulmonary Disease. Among the Blind, the One-Eyed Is King. American Journal of Respiratory and Critical Care Medicine, v. 174, n.8, p. 848-849, 2006.

MOORE, B.; BARRON. T.; JAMES, J.; SCOTT, J.; ROBINSON, K. (org.). Wiltshire Joint Strategic Needs Assessment (JSNA) for Wiltshire, 2009. Disponível em: <http://www.kirklees.gov.uk/community/statistics/jsna/jsna.shtml>. Acesso em: 12 dez. 2010. 